\section{Dynamic topographic mapping of the human bladder during voiding using functional near-infrared spectroscopy}

\author{
Andrew Macnab, ${ }^{a}$ Lynn Stothers, ${ }^{b, *}$ and \\ Babak Shadgan ${ }^{\mathrm{c}}$ \\ a University of British Columbia Hospital, Bladder Care \\ Centre, Room F329, 221 Wesbrook Mall, Vancouver, British \\ Columbia V6T 1 Z3 Canada \\ bUBC Hospital Room F329, 221 Wesbrook Mall, \\ Vancouver, British Columbia V6T 1 Z3 Canada \\ ${ }^{\mathrm{C}}$ University of British Columbia, Muscle Biophysics \\ Laboratory, VGH Research Pavilion, 611-828 West 10th \\ Avenue, Vancouver, British Columbia V5Z 1L8 \\ Canada
}

\begin{abstract}
Functional near-infrared spectroscopy (fNIRS) with multichannel instruments and grids of sourcedetector pairs can map regional change in oxygenation/ hemodynamics. Developed for cortical brain mapping, fNIRS technology has relevance in other organs where pathology affects the microcirculation. We describe fNIRS of the human bladder for evaluation of hemodynamic change during voiding. A $5 \times 5-\mathrm{cm}$ grid with two sourcedetector pairs is placed on the abdomen suprapubically in an asymptomatic male. In four separate trials, after natural bladder filling NIRS-derived changes in oxyhemoglobin $\left(\mathrm{O}_{2} \mathrm{Hb}\right)$, deoxyhemoglobin $(\mathrm{HHb})$, and total hemoglobin $(\mathrm{tHb})$ concentration are recorded during voiding (measured via uroflow), using four channels of a four wavelength continuous wave instrument. Graphic and video images (topographic mapping software) are generated. Changes in $\mathrm{tHb}$ occur following permission to void that predominantly reflected variation in $\mathrm{O}_{2} \mathrm{Hb}$; $\mathrm{tHb}$ peaks at maximum urine flow then falls to a nadir lasting to uroflow end. Change in fNIRS video color intensity correlates with graphic change in chromophore concentration. Color variations across the mapped area suggest regional hemodynamic variation. fNIRS bladder studies generate reproducible chromophore data consistent with single channel studies, but the dynamic color video and larger tissue area monitored potentially offer new methodology for investigating regional variations in bladder oxygenation and hemodynamics. @ 2009 Society of Photo-Optical Instrumentation Engineers. [DOI: 10.1117/1.3122886]
\end{abstract}

Keywords: functional near-infrared spectroscopy; detrusor; chromophore; deoxyhemoglobin; oxyhemoglobin; urodynamics; urology.

Paper 08460LR received Jan. 13, 2009; revised manuscript received Mar. 1, 2009; accepted for publication Mar. 4, 2009; published online Apr. 27, 2009.

*Address all correspondence to: Dr. Lynn Stothers, E-mail:
Functional near-infrared spectroscopy (fNIRS) with multichannel instruments and grids of source-detector pairs enables dynamic changes in regional cerebral blood flow $(\mathrm{CBF})$ to be mapped by measuring the concentration changes in cerebral hemoglobin. ${ }^{1-5}$ Detection of regional hemodynamic change also has relevance in other organs where pathology affects the functional state of the microcirculation. We report the feasibility of using fNIRS technology to generate dynamic color mapping of changes in oxygenated and deoxygenated hemoglobin in the human bladder during voiding.

The principles of NIRS and the ability of this noninvasive optical method to measure changes in tissue oxygenation and hemodynamics have been comprehensively reviewed. ${ }^{6,7}$ Urologic research using single channel continuous wave (cw) NIRS has investigated conditions with a hemodynamic or vascular etiology, and changes in chromophore concentration in the bladder wall muscle (detrusor) during voiding monitored transcutaneously via a suprapubic sensor. ${ }^{8}$ NIRS parameters are usually displayed graphically; composite graphs allow interpretation of the patterns and magnitude of change from baseline in relation to other measurements/events. Many systemic diseases causing vascular pathology negatively impact bladder function, probably through related changes in the organ's microcirculation affecting oxygen supply and demand. NIRS can detect physiologic changes in the bladder detrusor because of the physics of NIRS, the shallow depth of the anterior wall of the bladder below the abdominal wall (mean 2.8-cm range 1.24 to 3.94: unpublished data from 24 adults), its rich blood supply, and the hemodynamic and mechanical changes that occur. ${ }^{9-11}$ The rationale for confidence that NIRS contributes novel information on detrusor hemodynamics and oxygenation has been reviewed. ${ }^{8}$ Mechanical changes in the bladder wall and vasculature occur as the organ fills and empties, ${ }^{11}$ vessels must lengthen and shorten while maintaining organ perfusion, ${ }^{9}$ and blood flow falls during voiding. ${ }^{10}$ Voiding disorders become common with advancing age, consequently noninvasive optical diagnostic methodology with the capabilities of NIRS is attractive for investigation, and fNIRS has relevance for monitoring a larger area of the bladder and regional changes in the microcirculation.

Two fiber optic source-emitter pairs (optodes) of an Oxymon Mk III NIRS spectrometer (Artinis Medical Systems BV, Holland $)^{12}$ were configured in a four-channel four-point array with $5-\mathrm{cm}$ interoptode separation in a self-adhesive 5 $\times 5$-cm grid on the abdominal wall skin in the midline just superior to the symphysis pubis. Measurements on four different occasions captured four separate voiding cycles in a healthy 64 year-old male (no history of urologic symptoms) whose bladder filled naturally until urge to void was experienced. Ultrasound confirmed the location of the bladder below the optode grid. Baseline data were obtained using sequentially pulsed laser light at wavelengths of 766, 861, 906, and $971 \mathrm{~nm}$. The subject remained standing and voided normally into a uroflow meter (Laborie Medical Technologies Incorporated, Canada) to measure volume voided and peak rate of flow. During voiding, changes in the concentration of $\mathrm{O}_{2} \mathrm{Hb}, \mathrm{HHb}$, and total hemoglobin ( $\left.\mathrm{tHb}\right)$ were monitored continuously at $10 \mathrm{~Hz}$. The strong water signal $(975 \mathrm{~nm})$ was separated from the change of hemoglobin and was not part of

1083-3668/2009/14(2)/020507/3/\$25.00 @ 2009 SPIE 


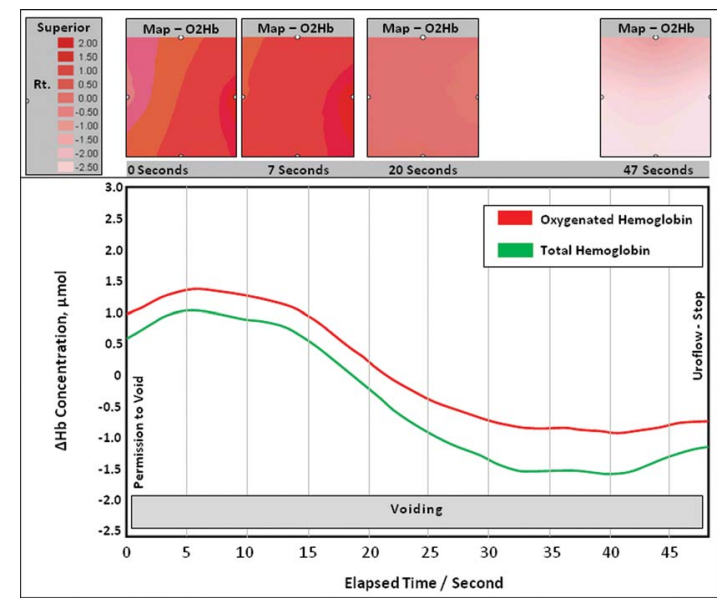

Fig. 1 fNIRS topographic images of $\mathrm{O}_{2} \mathrm{Hb}$ (oxyhemoglobin) in the detrusor muscle during voiding superimposed temporally on a graph of $\mathrm{O}_{2} \mathrm{Hb}$ and $\mathrm{tHb}$ concentration change from baseline. The graphs demonstrate that $\mathrm{O}_{2} \mathrm{Hb}$ is the predominant chromophore contributing to the overall change in total hemoglobin. This is reflected in the greater color variation in the fNIRS images during voiding as compared to $\mathrm{HHb}$ (Fig. 2). (Color online only.)

the fNIRS analysis. Changes from baseline values timed at permission to void were generated as line graphs and topographic video images from raw optical data using dedicated software (Oxysoft version 45, Artinis Medical Systems BV). The algorithm and 2-D plot computed reflect established principles related to the Beer-Lambert law and the back projection/linear interpolation scheme described by Franchesini et al.. ${ }^{13}$ No averaging is involved, and as no measurement of differential path length exists for the detrusor muscle, a value of 4 was used as for most NIRS muscle studies. All four voiding cycles were normal. Figures 1 and 2 and Video 1 of dynamic change are from cycle 1 , where voided volume was $410 \mathrm{ml}$, maximum flow was $22 \mathrm{ml}$, and residual volume was $10 \mathrm{ml}$.

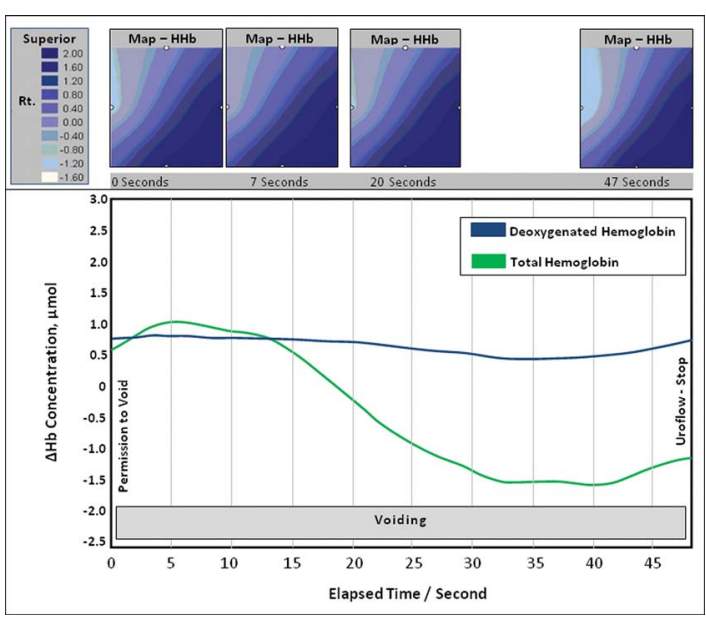

Fig. 2 fNIRS topographic images of $\mathrm{HHb}$ (deoxyhemoglobin) in the detrusor during voiding superimposed temporally on a graph of $\mathrm{HHb}$ and $\mathrm{tHb}$ concentration change from baseline. In comparison with Fig. 1, there is a much smaller contribution from $\mathrm{HHb}$ to the overall change in total hemoglobin. This is reflected by the small degree of variation in color change in the fNIRS images of chromophore concentration. (Color online only.)

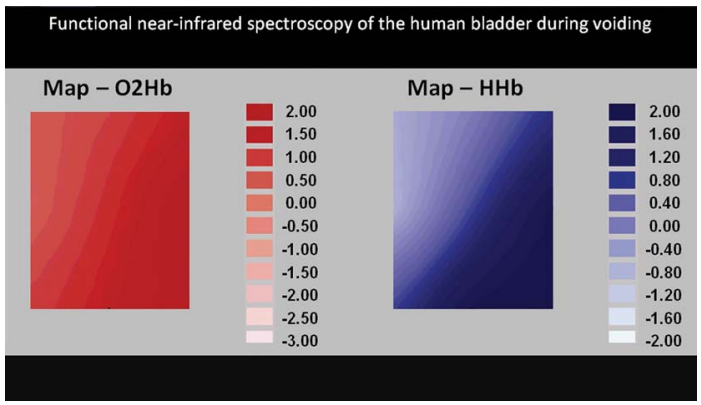

Video 1 fNIRS video sequences of dynamic color change of $\mathrm{O}_{2} \mathrm{Hb}$ (in red) beside $\mathrm{HHb}$ (in blue) from permission to void, through to peak urine flow, and on to the end of urine flow. The variations in color intensity predominantly reflect change in $\mathrm{O}_{2} \mathrm{Hb}$, and indicate an increase initially of the concentration of this chromophore in the detrusor as voiding begins, with a waning of this effect as the bladder empties. Fluctuating areas of localized change in color intensity are visible that have a wave-like pattern across the monitored field. These suggest regional hemodynamic changes within the detrusor as the bladder muscle contacts and the organ empties. (Color online only.) (MOV, 5 MB). [URL: http://dx.doi.org/10.1117/1.3122886.1].

The fNIRS video sequences have side-by-side displays of $\mathrm{O}_{2} \mathrm{Hb}$ and $\mathrm{HHb}$ concentration, (an example: voiding cycle 1 as shown in Video 1). The intensity of color change in $\mathrm{O}_{2} \mathrm{Hb}$ is greater than in $\mathrm{HHb}$ over the voiding cycle. This difference reflects the predominant contribution of $\mathrm{O}_{2} \mathrm{Hb}$ to the rise and fall in $\mathrm{tHb}$ that occurred. Figures 1 and 2 are composites of still images from the topographic video mapping superimposed onto traditional graphs of chromophore change. Each still color image is representative of the $5 \times 5-\mathrm{cm}$ area mapped at specific time points. Following permission to void, a brief initial rise in $\mathrm{O}_{2} \mathrm{Hb}$ occurred, evident in the video as an increase in color intensity (red), and on the line graphic as a change of $2.5 \mathrm{umol}$ from baseline. tHb peaked at maximum urine flow then fell to a nadir lasting to uroflow end. This decrease, seen in Video 1 as a waning in $\mathrm{O}_{2} \mathrm{Hb}$ color intensity, is evident in the still images that become paler, toward the completion of voiding. In addition, small fluctuating areas of localized regional change in color intensity are visible that have a wave-like pattern across the monitored field. These suggest that regional changes in $\mathrm{O}_{2} \mathrm{Hb}$ concentration occur within the detrusor as the bladder contracts and empties. The magnitude of change of these regional variations is 1 to 2 umol based on the scale (see figures) provided by the software.

This study demonstrates the feasibility of using an instrument with fNIRS capability to monitor the human bladder, and generate color video mapping of dynamic changes in $\mathrm{O}_{2} \mathrm{Hb}$ and $\mathrm{HHb}$ concentration occurring in the bladder wall during voiding. Prior phantom testing of the instrument used compared its linearity and noise performance against other devices. ${ }^{12}$ The instrument is sensitive and was the first to show activation changes in the motor cortex. ${ }^{14}$ The reproducible patterns of change in each chromophore monitored in all four voids lend some test-retest validity. Also, the chromophore changes observed only occurred during physiologic events related to voiding. The dynamic color changes seen on video correlate with conventional graphics of chromophore concentration generated from the same raw data. $\mathrm{O}_{2} \mathrm{Hb}$ constitutes the predominant component of the changes in $\mathrm{tHb}$ that occur, with minimal change evident in HHb. The same pattern 
of chromophore change is seen in voiding studies in normal humans using single channel NIRS. ${ }^{8}$ Traditional graphic display of NIRS data generated by robust algorithms reflects relative changes in blood volume and oxygenation within tissue, and allows several specific parameters to be measured. ${ }^{6,7}$ Studies using fNIRS provide insight into regional variation in cortical hemodynamics generated by neuronal activation in response to stimuli that increase $\mathrm{CBF} .{ }^{1-4,13,14}$ We hypothesize that fNIRS of the bladder as it contracts also monitors physiologic change in oxygenation and hemodynamics occurring within the microcirculation of the detrusor.

The application of fNIRS technology to the bladder has relevance, as a functional microvasculature is required to maintain tissue oxygenation under conditions of varying demand. In muscle this is achieved via the interplay of neuronal-, endothelial-, and erythrocyte-mediated mechanisms that initially alter the caliber of terminal arterioles to promote the local extraction of available oxygen, and then as metabolic demand increases, recruit intermediate and proximal arterioles. ${ }^{15}$ When systemic or regional pathology compromises the functional integrity of the microcirculation, symptoms of dysfunction occur. We recognize the potential limitation of using fNIRS to evaluate an organ that changes in size during measurement. However, fNIRS techniques using multiple source-detector pairs are less sensitive to movement than single channel systems, ${ }^{6}$ do not require strict motion restriction, ${ }^{4}$ and dynamic changes in regional CBF are reliably obtained during normal and pathological movements. ${ }^{1,3}$ Our subject voided without abdominal straining. The base of the bladder is predominantly interrogated because of the site of the source-detector grid. Consequently, as pelvic ultrasound suggests that voiding mainly generates alteration in size within the organ's dome, some change in bladder wall thickness within the field of measurement is more likely than frank movement. Importantly, the graphic and topographical changes in $\mathrm{O}_{2} \mathrm{Hb}$ and $\mathrm{HHb}$ detected via fNIRS differed significantly from one another, which suggests that they predominantly reflect physiologic change rather than an effect of movement. This premise is also supported by the proven diagnostic ability of NIRS bladder parameters, ${ }^{8,16}$ and animal data where single-channel NIRS yields comparable patterns of chromophore change from suprapubic transcutaneous monitoring and direct application of a NIRS source-detector behind the base of the surgically exposed bladder, where the absence of movement can be confirmed visually. ${ }^{17}$

The validity of the technique described, and the impression that fNIRS may show regional hemodynamic change in the detrusor, need to be clarified by further studies. However, the regional changes seen, which are particularly evident toward the completion of voiding, are compatible with what is known about the bladder's blood supply, ${ }^{9-11}$ and the response of a functional microcirculation to changes in oxygen supply and demand. $^{15}$

In urology, NIRS already adds previously unavailable information on bladder oxygenation and hemodynamics ${ }^{8}$ and a new noninvasive diagnostic capability. ${ }^{16}$ fNIRS could prove useful in both a research and clinical context, as local and systemic pathology can impact the microcirculation. Cerebral fNIRS studies suggest that variation in $\mathrm{tHb} / \mathrm{O}_{2} \mathrm{Hb}$ is the most sensitive indicator of alterations in energy demand and related fluctuations in regional blood flow, ${ }^{1,3,5}$ and that evoked blood flow responses differ in health and disease. ${ }^{5}$ Brain and bladder fNIRS share the ability to compare light intensity modulation in response to a physiologic stimulus (voiding in the case of the bladder) with baseline events free of stimulus. The fNIRS data in our subject's normal bladder show patterns of change in $\mathrm{tHb} / \mathrm{O}_{2} \mathrm{Hb}$ during voiding that closely resemble the change in normal brain during functional cortical activation. ${ }^{5}$ Further research in asymptomatic subjects is required to examine the reproducibility of bladder fNIRS; then investigation of symptomatic subjects could identify if specific trends in $\mathrm{tHb}$, $\mathrm{O}_{2} \mathrm{Hb}$, and $\mathrm{HHb}$ are associated with individual pathologies. The potential of fNIRS to contribute clinically in urology will depend on such research. NIRS optical techniques are attractive because of their noninvasive nature and proven ability to measure changes in tissue oxygenation and hemodynamics.

\section{References}

1. Y. Hoshi, "Functional near-infrared spectroscopy: current status and future prospects," J. Biomed. Opt. 12(6), 062106 (2007).

2. S. C. Bunce, M. Izzetoglu, K. Izzetoglu, B. Onaral, and K. Pourrezaei, "Functional near-infrared spectroscopy," IEEE Eng. Med. Biol. Mag. 25, 54-62 (2006)

3. T. Wilcox, H. Bortfeld, R. Woods, E. Wruck, and D. A. Boas, "Using near-infrared spectroscopy to assess neural activation during object processing in infants," J. Biomed. Opt. 10(1), 11010 (2005).

4. R. N. Aslin and J. Mehler, "Near-infrared spectroscopy for functional studies of brain activity in human infants: promise, prospects and challenges," J. Biomed. Opt. 10(1), 011009 (2005).

5. K. Sakatani, Y. Murata, N. Fujiwara, T. Hoshino, S. Nakamura, T. Kano, and Y. Katayama, "Comparison of blood-oxygen-dependent functional magnetic resonance imaging and near-infrared spectroscopy recording during functional brain activation in patients with stroke and brain tumors," J. Biomed. Opt. 12(6), 062100 (2007).

6. M. Wolf, M. Ferrari, and V. Quaresima, "Progress of near-infrared spectroscopy and topography for brain and muscle clinical applications," J. Biomed. Opt. 12(6), 062104 (2007).

7. M. Ferrari, L. Mottola, and V. Quaresima, "Principles, techniques and limitations of near infrared spectroscopy," Can. J. Appl. Physiol. 29(4), 463-487 (2004).

8. L. Stothers, B. Shadgan, and A. J. Macnab, "Urologic applications of near infrared spectroscopy," Can. J. Urol. 15(6), 4399-4409 (2008).

9. A. F. Barding, J. E. Greenland, I. W. Mills, G. McMurray, and S. Symes, "Blood supply to the bladder during filling," Scand. J. Urol. Nephrol., Suppl. 201, 25-31 (1999).

10. R. T. Kershen, K. M. Azadzoi, and M. B. Siroky, "Blood flow, pressure and compliance in the male human bladder," J. Urol. (Baltimore) 168, 121-25 (2002).

11. R. Kozlowski, M. B. Siroky, R. J. Krane, and K. M. Azadzoi, "Regulation of blood flow and microcirculation resistance in rabbit bladder," J. Urol. (Baltimore) 168, 1608-1614 (2002).

12. M. C. van der Sluijs, W. N. Colier, R. Houston, and B. Oeseburg, "A new and highly sensitive continuous wave near infrared spectrophotometer with multiple detectors," Proc. SPIE 3194, 63-72 (1997).

13. M. C. Franchesini, V. Toronov, M. E. Filiaci, E. Gratton, and S. Fantini, "On-line optical imaging of the human brain with 160-ms temporal resolution," Opt. Express 3(6), 49-57 (2000).

14. W. N.J.M Colier, V. Quaresima, G. Barattelli, P. Cavallari, M. Van der Sluijs, and M. Ferrari, "Detailed evidence of cerebral hemoglobin oxygenation changes in response to motor cortical activation revealed by a continuous wave spectrophotometer with $10 \mathrm{~Hz}$ temporal resolution," Proc. SPIE 2979, 390-396 (1998).

15. S. Segal, "Regulation of blood flow in the microcirculation," Microcirculation (Philadelphia) 12, 33-45 (2005).

16. L. Stothers, R. Guevara, and A. J. Macnab, "Classification of male lower urinary tract symptoms using mathematical modeling and a regression tree algorithm of non invasive near infrared parameters," Eur. Urol. (in press).

17. L. Stothers, B. Shadgan, and A. J. Macnab, "Near infra-red spectroscopy in the rabbit: response to hypoxia," J. Urol. (Baltimore) (in press). 